\section{Flower power}

The root and stem bark of magnolia is a traditional Chinese and Japanese medicine, and an active component of this plant, called honokiol, has anti-tumour activity in laboratory models. Kenneth Anderson and colleagues have now deduced the mechanism of action of honokiol and shown that it can overcome drug resistance in multiple-myeloma cells.

Honokiol inhibited the growth of multiple-myeloma cell lines and tumour cells isolated from patients with relapsed refractory multiple myeloma, but it had no inhibitory effect on normal peripheral blood mononuclear cells. Most drugs that are used to treat multiple myeloma induce tumour cell death by activating caspases and, indeed, the authors showed that honokiol also induces apoptosis by a caspase-dependent pathway. However, apoptosis induction was only partially blocked by the pan-caspase inhibitor z-VAD-fmk, so the authors looked for evidence of caspase-independent activation. They found that honokiol induced the release of apoptosis-inducing factor from mitochondria, which activates caspase-independent cell death.

Importantly, honokiol induced apoptosis even in myeloma cells that are resistant to drugs often used in the treatment of myeloma. In addition, combined treatment of multiple-myeloma cells with honokiol and the proteasome inhibitor bortezomib increased the inhibition of tumour cell growth. The authors postulate that this is due to modulation by honokiol of the expression of heat-shock proteins, which are usually stimulated by bortezomib and can lead to resistance to bortezomib-induced apoptosis. Honokiol also overcomes another source of drug resistance in multiple myeloma - the production in the bone marrow of interleukin- 6 and insulin-like growth factor, both of which promote tumour growth. Clinical studies to test the effectiveness of honokiol in relapsed refractory multiple myeloma are warranted.

Ezzie Hutchinson

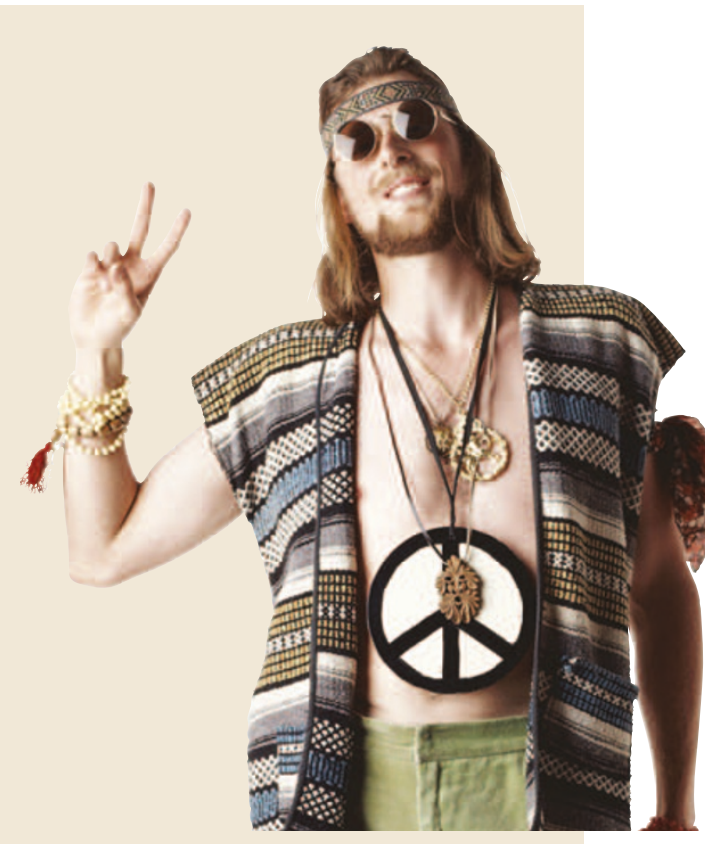

(9) References and links

ORIGINAL RESEARCH PAPER Ishitsuka, K. et al. Honokiol overcomes conventional drug resistance in human multiple myeloma by induction of caspase-dependent and

-independent apoptosis. Blood 106, 1794-1800 (2005) WEB SITE

Kenneth Anderson's lab: http://www.dana-farber.org/res/ physician/detail.asp?personID=5\&RD=True\&group= (Clinician+and+Researcher)

\title{
Lung cancer suppressors
}

Deletions of chromosomes $3 p$ and $1 p$ are among the most frequently observed mutations in human lung cancer. However, the candidate genes in these regions that have been studied to date have not turned out to be tumour suppressors in mouse models. Now, Hollander

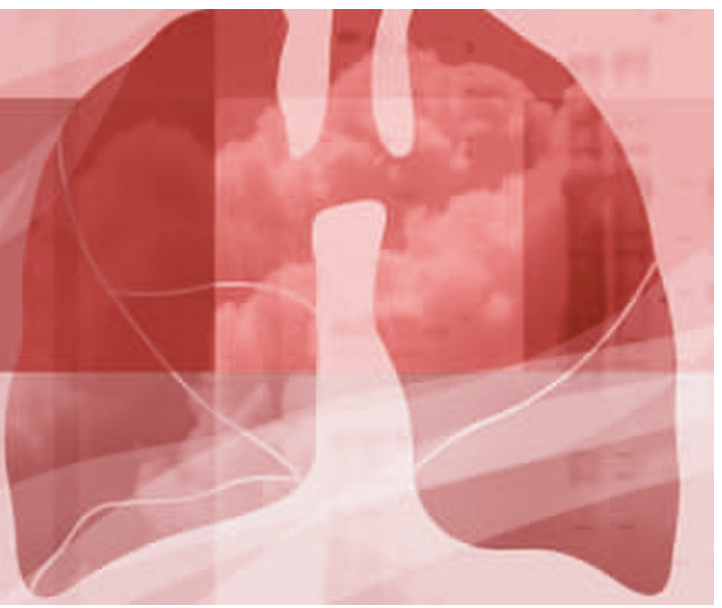

et al. have shown that deletion of Xpc causes lung tumours in mice, and also that Gadd45a deletions promote tumour progression.

$X P C$ and GADD45A are found in humans at $3 p$ and $1 p$, respectively, and both are involved in nucleotide excision repair. XPC polymorphisms in humans have been linked to the risk of developing cancer, and loss-of-function mutations of XPC cause skin cancer in the context of xeroderma pigmentosum. On this basis, the researchers studied the incidence of lung cancer over 24 months in mice that were homozygous for either Xpc or Gadd45a, as well as double mutants and controls. Mice were killed when they became ill or at 24 months if they survived until then. Lung tumours were then counted and graded.

All of the $X \mathrm{pc}^{-/-}$mice developed lung adenomas, although few showed progression to adenocarcinoma. By contrast, the controls and the Gadd $45 a^{-/-}$mice showed no evidence of tumour formation. In the double mutant, most tumours progressed to adenocarcinomas. These results show that $X p c$ is a tumour suppressor but that its loss is not sufficient for progression. Gadd45a, on the other hand, is involved in preventing progression.

The researchers also looked for other factors that are known to correlate with lung cancer. Polymorphisms in other markers of susceptibility, such as Pas 1 and the Par genes, did not correlate with the incidence of tumours in these mice. Also, the tumours did not show consistent mutation in KRas, which was thought to be a probable result of $X p c$ inactivation.

Studies of human lung cancers show that the markers that flank XPC on chromosome $3 p$ are lost in most tumours. The markers flanking GADD 45A on chromosome 1p, on the other hand, are only lost in some lung tumours but are also lost in many other tumour types.

Tobacco carcinogens are the main cause of lung cancer. The discovery of a lung-tumoursuppressor gene that might repair the DNA damage caused by tobacco carcinogens gives a fresh insight into the mechanisms of lung carcinogenesis.

Patrick Goymer

\section{9) References and links}

ORIGINAL RESEARCH PAPER Hollander, M. C. et al. Deletion of XPC leads to lung tumors in mice and is associated with early events in human lung carcinogenesis. Proc. Natl Acad. Sci. USA 2 September 2005 (doi:10.1073/pnas.0503133102) 